\title{
Duloxetine for the prevention of oxaliplatin induced peripheral neuropathy: a randomized, placebo- controlled, double-blind clinical trial
}

Mahdi Aghili

Tehran University of Medical Sciences

Nima Mousavi Darzikolaee ( $\nabla$ mousavi.nima@yahoo.com )

Tehran University of Medical Sciences

Mohammad Babaei

Tehran University of Medical Sciences

Reza Ghalehtaki

Tehran University of Medical Sciences

Farshid Farhan

Tehran University of Medical Sciences

Seyede Zahra Emami Razavi

Tehran University of Medical Sciences

Saeed Rezaei

Tehran University of Medical Sciences

Ebrahim Esmati

Tehran University of Medical Sciences

Farhad Samiei

Tehran University of Medical Sciences

Mohadese Azadvari

Tehran University of Medical Sciences

Borna Farazmand

Tehran University of Medical Sciences

Ardavan Amiri

Tehran University of Medical Sciences

\section{Research Article}

Keywords: Oxaliplatin, Peripheral Neuropathy, Duloxetine, Prevention

Posted Date: March 24th, 2021

DOI: https://doi.org/10.21203/rs.3.rs-353734/v1 
License: (c) (i) This work is licensed under a Creative Commons Attribution 4.0 International License. Read Full License

Version of Record: A version of this preprint was published at Journal of Gastrointestinal Cancer on April 14th, 2022. See the published version at https://doi.org/10.1007/s12029-022-00824-0. 


\section{Abstract}

Introduction

Peripheral neuropathy is a dose-limiting adverse effect of oxaliplatin. The aim of this study was to evaluate the efficacy and safety of duloxetine in the prevention of oxaliplatin-induced peripheral neuropathy (OIPN).

Method

Cancer patients receiving oxaliplatin chemotherapy were randomized into two arms. Duloxetine $60 \mathrm{mg}$ capsule was given in the first 14 days of each chemotherapy cycle to one arm and placebo was similarly given to another arm. We compared the two arms based on the incidence of neuropathy and the results of the nerve conduction study.

Results

Thirty two patients were randomized to duloxetine and placebo arms. Most of the patients had rectal cancer (90.6\%). Compared with the placebo arm, patients in the duloxetine arm had a lower percentage of chemotherapy cycles (mean) in which they reported distal paresthesia ( $84 \%$ vs. $51 \%, P=0.01)$ and throat discomfort $(69 \%$ vs. $37 \%, P=0.01)$. There was no difference in the percentage of cycles in which patients reported cold-induced dysesthesia. Highest grade of neuropathy in each cycle was not significantly different between the two arms. Six weeks after the last cycle of chemotherapy, nerve conduction velocity was significantly higher in duloxetine arm compared to the placebo arm in the deep peroneal nerve and tibial nerve. Duloxetine was safe and well-tolerated.

Conclusion

In spite of small sample size, results of this study suggests potential efficacy of duloxetine in the prevention of OIPN, as indicated by objective measures of neurotoxicity and some patient-reported symptoms.

\section{Introduction}

Chemotherapy-Induced Peripheral Neuropathy (CIPN) is a well-known side effect of chemotherapy affecting up to $48 \%$ of cancer patients who receive multiple chemotherapeutic agents [1]. By increasing the number of cancer survivors receiving chemotherapy, this complication represents a significant problem affecting physical, emotional, and cognitive functions in cancer survivors [2]. Chemotherapy agents with higher incidence of CIPN are platinum, taxanes, vinca alkaloids, and bortezomib [3].

Oxaliplatin is a third-generation platinum. OIPN remains a treatment-limiting factor and can be divided into two types of neuropathy, acute and chronic [4]. Acute OIPN is transient and occurs in the majority of patients (85-95\%), but chronic OIPN can persist for months or years and occurs in $10-20 \%$ of patients. 
Chronic OIPN has been reported to be dose-dependent with severe symptoms occurring at the cumulative dose above $750-850 \mathrm{mg} / \mathrm{m}^{2}[2,5]$. Approximately two-thirds of patients will have symptoms one-year post-treatment or beyond $[1,6]$. Typical presentations of OIPN are paresthesia (tingling) in hands and feet, cold-induced neuropathy, throat discomfort, and muscle cramps [7].

Several pharmacological agents such as amitriptyline, nortriptyline, and venlafaxine have been studied for treatment and prevention of CIPN with limited success. Duloxetine, a serotonin and norepinephrine (NE) reuptake inhibitor, is an antidepressant drug that has shown some efficacy in treating painful CIPN induced by oxaliplatin and paclitaxel $[8,9]$. The American Society of Clinical Oncology (ASCO) suggested duloxetine as the only treatment for CIPN (moderate recommendation) in 2020, while no agents was recommended for the prevention of CIPN $[10,11]$.

Considering the efficacy of duloxetine in the treatment of CIPN, we aimed to evaluate prophylactic effect of duloxetine in CIPN in cancer patients receiving oxaliplatin based chemotherapy as well.

\section{Patients And Methods}

\section{Study Design}

This randomized double-blinded, placebo-controlled phase $\Pi$ clinical trial was performed in radiation oncology ward at the Iran cancer institute (Tehran University of medical sciences, Tehran, Iran) from December 2016 to March 2019. The study was registered in the Iranian registry of clinical trials (Trial registration code: IRCT20170211032494N2). This study was performed in line with the principles of the Declaration of Helsinki and approved by institutional review board and ethical committee.

\section{Patients}

Eligible patients were those with pathologically confirmed colorectal and esophageal cancer patients, aged 18-75, candidate for receiving CAPOX (capecitabine-oxaliplatin) regimen, and good performance status based on Eastern Cooperative Oncology Group (ECOG 0-1) performance scale. Exclusion criteria were history of prior therapy with oxaliplatin, previous cancer except non-metastatic non-melanoma skin cancer, diabetes mellitus, any kind of neuropathy, chronic renal or liver disease, and Concomitant medications that may cause (e.g., certain HIV drugs) or prevent neuropathy (e.g., venlafaxine). Informed consent in written format was obtained from all of the patients participating in the trial.

\section{Chemotherapy Protocol}

CAPOX regimen was given with standard dosage (capecitabine $1000 \mathrm{mg} / \mathrm{m}^{2} \mathrm{BD}$ and oxaliplatin 130 $\mathrm{mg} / \mathrm{m}^{2}$ ) every 21 days. Oxaliplatin was diluted in 500 milliliters of glucose $5 \%$ and was infused over 2 hours.

\section{Randomization and Intervention}


We randomized the eligible patients into two arms using randomly assigned permuted blocks method. The intervention arm received duloxetine on the first 14 days of each chemotherapy cycle (first dose was given 1 hour before chemotherapy administration). The Control arm received similarly appearing placebo capsules in the same manner. Duloxetine and placebo capsules were manufactured by the same pharmaceutical company. Neither the physicians nor the patients were aware of the type of drug.

\section{Outcome Assessment}

The primary objective of this study was to assess the incidence of neuropathy, both subjectively and objectively. The secondary objective was the safety of duloxetine. For subjective evaluation of acute OIPN, patients were visited one day before and within one week after each oxaliplatin administration, and they were questioned by a physician about the presence of paresthesia of the hands and feet (distal paresthesia), cold dysesthesia or cold-induced neuropathy (touching cold or drinking cold liquid), and throat discomfort. Patients were also visited 6 weeks after the last course of chemotherapy for assessment of chronic OIPN. Grade of neuropathy was recorded according to CTCAE4 (Common Terminology Criteria for Adverse Events version 4). For objective evaluation, before initiating oxaliplatin (baseline) nerve conduction studies (NCS) was done for sensory (sural) and motor nerves (deep peroneal and tibial). This assessment was repeated 6 weeks after the completion of chemotherapy. All the NCS were carried out by one expert specialist to avoid inter-observer bias. To monitor the side effects of duloxetine, patients were also asked about the presence of fatigue, dizziness, somnolence, and other events.

\section{Statistical Analysis}

According to a previous pilot study [12] $63.3 \%$ improvement in subjective OIPN and $47.4 \%$ improvement in objective grade of OIPN was seen in patients who received duloxetine. Thus considering the incidence of acute OIPN (about $90 \%$ ) $[5,10,13]$ with $80 \%$ power and $10 \%$ drop-out rate we needed a maximum 22 subjects in each arm. Analyses were conducted to compare the two arms in each cycle and also entirely. Relative frequency of neuropathy in each cycle was compared between the two arms using the chisquared test. Independent sample t-test was conducted to compare proportion of chemotherapy cycles (mean) in which patients reported neuropathy. We opted the ANOVA for repeated measures to compare the NCS parameter, such as velocity before and after the intervention between arms. A $P$-value $<0.05$ was considered statistically significant. Data were analyzed with SPSS for Windows version 21.0 (SPSS Inc., Chicago, IL).

\section{Results}

\section{Clinical Characteristics of Patient}

Forty patients were assessed for eligibility. After the exclusion of 8 patients, 32 were considered for the intervention, 17 in the duloxetine arm and 15 in the placebo arm (figure 1). 
The general characteristics of the patients were comparable in the two arms (table 1). Median number of chemotherapy cycles was four. At baseline assessment, none of the patients complained of the paresthesia, throat discomfort, and cold-induced neuropathy. Diagnostic test results of amplitude and velocity in sural, deep peroneal and tibial nerves were comparable in the two arms as well (Table 7).

\section{Duloxetine Efficacy}

Ninety percent of the patients (29 out of 32 ) suffered from at least one kind of acute OIPN symptoms such as distal paresthesia, throat discomfort, or cold dysesthesia in at least one chemotherapy cycle; only three patients did not complain of any symptoms of OIPN, all in duloxetine arm. In contrast, patient experiencing the most severe neurotoxicity (grade 2 in two out of three courses of chemotherapy) was in the placebo arm. The incidence of neuropathy was compared in each cycle and also entirely between the two arms. Frequency of patient-reported symptoms in almost all cycles was lower in the duloxetine arm compared to placebo arm, although the difference was not significant except for throat discomfort in cycle 4 (Table 2). Grade of neuropathy based on CTCAE in each cycle and also 6 weeks after last cycle was not significantly different between the two arms (table 3). Percentage of chemotherapy cycles (mean) in which patients reported distal paresthesia and throat discomfort was significantly lower in duloxetine arm compared to placebo arm $(P=0.01)$ but there was no difference in the percentage of cycles in which patients reported cold-induced dysesthesia $(P=0.13)$ (Table 4).

NCV in second NCS in deep peroneal and tibial nerves were significantly higher in duloxetine arm compared to placebo arm in favor of duloxetine efficacy (Table 5). There was no significant difference in terms of sensory nerve action potential amplitude in the examined nerve between the two arms before and after treatment.

\section{Duloxetine Side Effects}

Duloxetine was safe and it was tolerated well. None of the patients required a temporary or permanent discontinuation of duloxetine due to adverse effects. The most frequent side effects were nausea, somnolence, dry mouth, and dizziness which were not significantly different between the two arms (Table 6). There were no events of grade 3 or 4 adverse effect.

\section{Other findings}

After a median follow up of 30 months, 22 patients out of 32 were alive; 14 in duloxetine arm and 8 in placebo arm. Rate of neuropathy was $40.9 \%$ totally (all grade 1). Five patients in duloxetine arm (35.7\%) and four patients in placebo arm (50\%) suffered from long term OIPN. Most of long term neuropathy was in form of paresthesia in foot.

\section{Discussion}

Several clinical trials have been conducted for the treatment of CIPN, but not much success has been achieved $[5,10]$. Given the lack of an effective treatment, investigating prophylactic strategies might be of 
value; such a policy showed success in chemotherapy-induced nausea and vomiting [14]. Most of the previous trials in the prevention of CIPN failed to find an effective neuroprotective agent [15-17], although there are a few positive studies too [18]. Different drugs including Calcium and magnesium, Pregabalin, Metformin, Venlafaxine, and Vitamin E has been used for the prevention of OIPN, yet there is no recommendation for daily practice [19-24].

To the best of our knowledge this is the first randomized clinical trial to evaluate efficacy of duloxetine in the prevention of CIPN. Results of this study suggests potential efficacy of duloxetine.

We found three clinical trials in which duloxetine were used for treating CIPN. The first one was a pilot study in 39 colorectal cancer patients suffering from CIPN. Duloxetine was effective and tolerable and the authors suggested that "In the future, it may play a role in effectively treating chronic OIPN" [12]. The second one was a high quality randomized clinical trial that led to 2014 American Society of Clinical Oncology's (ASCO) moderate recommendation on duloxetine as the only drug for the treatment of CIPN. They evaluated 231 patients with CIPN (due to taxane or platinum) randomized to two groups of duloxetine ( $30 \mathrm{mg} /$ day in the first week and then continued by $60 \mathrm{mg} /$ day) and placebo. They concluded that among patients with painful CIPN, duloxetine for 5 weeks resulted in a greater reduction in pain intensity compared to placebo [9]. The third study was held in Japan on 34 patients who had received oxaliplatin, paclitaxel, vincristine, or bortezomib. Duloxetine for 4 weeks, at the standard dose in japan ( $20 \mathrm{mg} /$ day for one week and then increased to $40 \mathrm{mg} /$ day), was associated with improved neuropathy [25].

We planned to start duloxetine at the same dosage as the previous studies but since at the time of study, duloxetine capsule $30 \mathrm{mg}$ was not easily available, we started with $60 \mathrm{mg} /$ day of duloxetine. Acute OIPN occurred in 90 percent of the patients which is consistent with previous studies $[5,10,13]$. In this study some objectives were improved and some were not. Interpretation of these results should be done cautiously. Relative frequency of distal paresthesia, throat discomfort, and cold induced neuropathy in each cycle was non-significantly reduced in duloxetine arm compared to placebo arm. Proportion of chemotherapy cycles (mean) in which patients reported symptoms of neuropathy was significantly lower in two out of three measured symptoms (distal paresthesia and throat discomfort) in duloxetine arm. The study did not detect an improvement in the clinician assessment of grade of neuropathy via CTCAE perhaps due to sample size and low oxaliplatin cumulative dose. Most of the patients in this study did not receive high cumulative doses of oxaliplatin (i.e. $>750-850 \mathrm{mg} / \mathrm{m}^{2}$ ), so the severity of OIPN in both groups was low (no Grade 3 of neuropathy).

In terms of objective assessment, results of the nerve conduction velocity in the tibial and deep peroneal nerves were in favor of duloxetine efficacy. Reduction in sensory nerve action potential (SNAP) amplitudes is also an expected finding of chronic CIPN due to axonal injury, but we did not find significant difference in terms of SNAP amplitude between the two arms. In an interesting study it was shown that the SNAP amplitude did not significantly change until after 21-24 weeks of oxaliplatin-based chemotherapy initiation (8/9 cycles of chemotherapy) while in our study most of the patients were 
examined in $<21$ weeks of chemotherapy initiation ( $4 / 5$ cycles of chemotherapy), so one explanation may be inappropriate time interval or low oxaliplatin cumulative dose [26].

Adverse effects of duloxetine were tolerable. In contrast to previous studies which prescribed duloxetine continuously, we prescribed duloxetine intermittently (two weeks on and one week off) which might explain lower rate of duloxetine discontinuation in this study. None of the patients experienced serotonin syndrome, which has been mentioned as a concern due to the potential interaction of duloxetine and other drugs that inhibit serotonin reuptake (i.e., Granisetron that was used as a premedication drug before chemotherapy) [27].

The most common cited mechanism of acute OIPN is related to the chelation of calcium by oxalate [28], but this is not relevant to the known mechanism of duloxetine effects. In a recent study, duloxetine was shown to be neuroprotective in OIPN, both in vitro and in vivo. Inhibiting the MAPK signaling and consequently preventing the NF-kB activation was considered as the duloxetine mechanism of action in one study [29]. In an animal research anti-allodynia effect of duloxetine in OIPN was shown to be mediated by the spinal alpha1-adrenergic receptors [30]. Norepinephrine (NE) and serotonin inhibit the transmission of pain signals into Dorsal Root Ganglia (DRG) [31]. Considering duloxetine effect on inhibition of NE and serotonin reuptake, it was thought to be useful for chronic neuropathy like diabetic peripheral neuropathy which may explains the potential efficacy of duloxetine in chronic CIPN as well.

\section{Limitation}

The findings of this study have to be seen in light of some limitations. The first one is small sample size, so the study might not have enough power to demonstrate duloxetine efficacy in all endpoints. The second one is the difference in oxalipaltin cumulative dose in the two groups. The third one is relatively short duration of chemotherapy administration in this trial, so that most of the patients did not receive a high cumulative dose of oxaliplatin.

\section{Conclusion}

It's challenging to determine whether this study did or did not prove efficacy of duloxetine since some endpoints were improved and others were not, but this small study suggests potential efficacy of duloxetine in the prevention of OIPN, as indicated by objective measures of neurotoxicity and some patient reported symptoms. Larger randomized trials are necessary to confirm efficacy of duloxetine in the prevention of OIPN.

\section{Declarations}

Ethical approval statements: This study was performed in line with the principles of the Declaration of Helsinki and approved by institutional review board and ethical committee. 
Funding and conflict of interest statement: The authors whose names are listed above declare that there is no conflict of interest regarding the publication of this article.

Data availability statement: The data that support the findings of this study are available from the corresponding author upon reasonable request.

\section{Acknowledgment}

This study was a part of post graduate thesis supported by Tehran university of medical sciences (grant number: 9311282025).

\section{Conflict of interest}

The authors declare that they have no conflict of interest.

\section{References}

1. Seretny M, Currie GL, Sena ES, et al. Incidence, prevalence, and predictors of chemotherapy-induced peripheral neuropathy: A systematic review and meta-analysis. PAIN®. 2014;155:2461-70.

2. Mols F, Beijers T, Lemmens V, et al. Chemotherapy-induced neuropathy and its association with quality of life among 2-to 11-year colorectal cancer survivors: results from the population-based PROFILES registry. J Clin Oncol. 2013;31:2699-707.

3. Pachman DR, Barton DL, Watson JC, et al. Chemotherapy-Induced Peripheral Neuropathy: Prevention and Treatment. Clin Pharmacol Ther. 2011;90:377-87.

4. Gamelin E, Gamelin L, Bossi L et al, editors. Clinical aspects and molecular basis of oxaliplatin neurotoxicity: current management and development of preventive measures. Semin Oncol; 2002.

5. Park SB, Goldstein D, Krishnan AV, et al. Chemotherapy-induced peripheral neurotoxicity: A critical analysis. CA Cancer J Clin. 2013;63:419-37.

6. Beijers AJM, Mols F, Vreugdenhil G. A systematic review on chronic oxaliplatin-induced peripheral neuropathy and the relation with oxaliplatin administration. Support Care Cancer. 2014;22:19992007.

7. Grothey A, Nikcevich DA, Sloan JA, et al. Intravenous calcium and magnesium for oxaliplatin-induced sensory neurotoxicity in adjuvant colon cancer: NCCTG N04C7. J Clin Oncol. 2011;29:421.

8. Okuma K, Shiraishi K, Kanai Y, et al. Improvement in quality of life by using duloxetine for chemotherapy-induced peripheral neuropathy (CIPN): a case report. Support Care Cancer. 2016;24:4483-5.

9. Smith E, Pang H, Cirrincione $\mathrm{C}$, et al. Effect of duloxetine on pain, function, and quality of life among patients with chemotherapy-induced painful peripheral neuropathy: a randomized clinical trial. Jama. 2013;309:1359-67. 
10. Hershman DL, Lacchetti C, Dworkin RH, et al. Prevention and management of chemotherapy-induced peripheral neuropathy in survivors of adult cancers: American Society of Clinical Oncology clinical practice guideline. J Clin Oncol. 2014;32:1941-67.

11. Loprinzi CL, Lacchetti C, Bleeker J, et al. Prevention and management of chemotherapy-induced peripheral neuropathy in survivors of adult cancers: ASCO guideline update. J Clin Oncol. 2020;38:3325-48.

12. Yang Y-H, Lin J-K, Chen W-S, et al. Duloxetine improves oxaliplatin-induced neuropathy in patients with colorectal cancer: an open-label pilot study. Support Care Cancer. 2012;20:1491-7.

13. Argyriou AA, Cavaletti G, Briani C, et al. Clinical pattern and associations of oxaliplatin acute neurotoxicity. Cancer. 2013;119:438-44.

14. Hesketh PJ. Chemotherapy-Induced Nausea and Vomiting. N Engl J Med. 2008;358:2482-94.

15. Afonseca SOd, Cruz FM, Cubero DdIG, et al. Vitamin E for prevention of oxaliplatin-induced peripheral neuropathy: a pilot randomized clinical trial. Sao Paulo Medical Journal. 2013;131:35-8.

16. Albers JW, Chaudhry V, Cavaletti G, et al (2014) Interventions for preventing neuropathy caused by cisplatin and related compounds. Cochrane Database of Systematic Reviews.

17. De Andrade DC, Teixeira MJ, Galhardoni R, et al. Pregabalin for the Prevention of Oxaliplatin-Induced Painful Neuropathy: A Randomized, Double-Blind Trial. Oncologist. 2017;22:1154.

18. Aghili M, Zare M, Mousavi N, et al. Efficacy of gabapentin for the prevention of paclitaxel induced peripheral neuropathy: A randomized placebo controlled clinical trial. Breast J. 2019;25:226-31.

19. Cavaletti G. Calcium and magnesium prophylaxis for oxaliplatin-related neurotoxicity: is it a trade-off between drug efficacy and toxicity? Oncologist. 2011;16:1667.

20. Loprinzi CL, Qin R, Dakhil SR, et al. Phase III randomized, placebo-controlled, double-blind study of intravenous calcium and magnesium to prevent oxaliplatin-induced sensory neurotoxicity (N08CB/Alliance). J Clin Oncol. 2014;32:997.

21. El-Fatatry BM, Ibrahim OM, Hussien FZ, et al. Role of metformin in oxaliplatin-induced peripheral neuropathy in patients with stage III colorectal cancer: randomized, controlled study. Int J Colorectal Dis. 2018;33:1675-83.

22. Huang H, He M, Liu L, et al. Vitamin E does not decrease the incidence of chemotherapy-induced peripheral neuropathy: a meta-analysis. Contemporary Oncology. 2016;20:237.

23. Jordan B, Jahn F, Beckmann J, et al. Calcium and magnesium infusions for the prevention of oxaliplatin-induced peripheral neurotoxicity: a systematic review. Oncology. 2016;90:299-306.

24. Zimmerman C, Atherton PJ, Pachman D, et al. MC11C4: a pilot randomized, placebo-controlled, double-blind study of venlafaxine to prevent oxaliplatin-induced neuropathy. Support Care Cancer. 2016;24:1071-8.

25. Hirayama Y, Ishitani K, Sato Y, et al. Effect of duloxetine in Japanese patients with chemotherapyinduced peripheral neuropathy: a pilot randomized trial. International Journal of Clinical Oncology. 2015;20:866-71. 
26. Lehky T, Leonard G, Wilson R, et al. Oxaliplatin-induced neurotoxicity: acute hyperexcitability and chronic neuropathy. Muscle Nerve. 2004;29:387-92.

27. Spina E, Trifirò G, Caraci F. Clinically Significant Drug Interactions with Newer Antidepressants. CNS Drugs. 2012;26:39-67.

28. Grolleau F, Gamelin L, Boisdron-Celle M, et al. A Possible Explanation for a Neurotoxic Effect of the Anticancer Agent Oxaliplatin on Neuronal Voltage-Gated Sodium Channels. J Neurophysiol. 2001;85:2293-7.

29. Meng J, Zhang Q, Yang C, et al (2019) Duloxetine, a Balanced Serotonin-Norepinephrine Reuptake Inhibitor, Improves Painful Chemotherapy-Induced Peripheral Neuropathy by Inhibiting Activation of p38 MAPK and NF-KB. Frontiers in Pharmacology 10.

30. Kim W, Chung Y, Choi S, et al. Duloxetine protects against oxaliplatin-induced neuropathic pain and spinal neuron hyperexcitability in rodents. Int J Mol Sci. 2017;18:2626.

31. Willis W, Westlund K. Neuroanatomy of the pain system and of the pathways that modulate pain. $J$ Clin Neurophysiol. 1997;14:2-31.

\section{Tables}

Table 1 Patients' characteristics

\begin{tabular}{|c|c|c|c|}
\hline Characteristic & Placebo arm $\mathrm{n}=15$ & Duloxetine arm $\mathrm{n}=17$ & P value \\
\hline Gender (female) & $3(20 \%)$ & $5(29 \%)$ & 0.5 \\
\hline Age (year), Mean & $55 \pm 11.6$ & $53 \pm 12.4$ & 0.4 \\
\hline \multicolumn{4}{|l|}{ Tumor type } \\
\hline Rectum & $13(87 \%)$ & 16 (94\%) & \\
\hline Esophagus & $2(13 \%)$ & $0(0 \%)$ & 0.4 \\
\hline Colon & $0(0 \%)$ & $1(6 \%)$ & \\
\hline Cumulative oxaliplatin dose $\left(\mathrm{mg} / \mathrm{m}^{2}\right)$, Mean & $420.6 \pm 202$ & $570.5 \pm 178$ & 0.03 \\
\hline Metastatic Disease & $4(27 \%)$ & $2(12 \%)$ & 0.3 \\
\hline
\end{tabular}

Table 2 Frequency of patients' reported symptoms of neuropathy in each cycle in the two groups 


\begin{tabular}{|c|c|c|c|c|c|}
\hline & & Cycle 1 & Cycle 2 & Cycle 3 & Cycle 4 \\
\hline \multirow[t]{2}{*}{ Number of patients } & Duloxetine & 17 & 16 & 15 & 15 \\
\hline & Placebo & 15 & 12 & 11 & 8 \\
\hline \multicolumn{6}{|l|}{ Paresthesia } \\
\hline & Duloxetine & $47.1 \%(8)$ & $62.5 \%(10)$ & $53.3 \%(8)$ & $53.3 \%(8)$ \\
\hline & Placebo & $73.3 \% \quad(11)$ & $83.3 \%$ & $81.8 \% \quad(9)$ & $75 \% \quad(6)$ \\
\hline \multirow[t]{2}{*}{ Throat discomfort } & Duloxetine & $35.3 \%(6)$ & $43.8 \%(7)$ & $40.0 \%(6)$ & $26.7 \%(4)$ \\
\hline & Placebo & $53.3 \% \quad(8)$ & $58.3 \%$ & $63.6 \%$ & $75.0 \%(6) *$ \\
\hline \multirow[t]{2}{*}{ Cold-induced dysesthesia } & Duloxetine & $52.9 \% \quad(9)$ & $68.8 \% \quad(11)$ & $66.7 \%(10)$ & $66.7 \%(10)$ \\
\hline & Placebo & $66.7 \%(10)$ & $66.7 \%(8)$ & $81.8 \%$ (9) & $87.5 \%(7)$ \\
\hline
\end{tabular}

*P value: 0.02

Table 3 Grade of neuropathy in each cycle in the two groups

\begin{tabular}{llclll}
\hline & & Number of patients & Grade 1 & Grade 2 & P value \\
\hline Cycle 1 & Duloxetine & 17 & $64.7 \%(11)$ & $0 \%(0)$ & 0.23 \\
& Placebo & 15 & $80.0 \%(12)$ & $6.7 \%(1)$ & \\
Cycle 2 & Duloxetine & 16 & $75.0 \%(12)$ & $6.3 \%(1)$ & 0.89 \\
& Placebo & 12 & $66.7 \%(8)$ & $8.3 \%(1)$ & \\
Cycle 3 & Duloxetine & 15 & $66.7 \%(10)$ & $0 \% \quad(0)$ & 0.09 \\
& Placebo & 11 & $54.5 \%(6)$ & $27.3 \%(3)$ & \\
Cycle 4 & Duloxetine & 15 & $66.7 \%(10)$ & $6.7 \%(1)$ & 0.69 \\
& Placebo & 8 & $75.0 \%(6)$ & $12.5 \%(1)$ & \\
Six weeks after & Duloxetine & 17 & $47.1 \%(8)$ & $5.9 \%(1)$ & 0.40 \\
last cycle & Placebo & 13 & $69.2 \%(9)$ & $7.7 \%(1)$ & \\
\hline
\end{tabular}

Table 4 Overall subjective neuropathy 


\begin{tabular}{|c|c|c|c|c|}
\hline & & $\begin{array}{c}\text { Placebo } \\
n=15\end{array}$ & $\begin{array}{c}\text { Duloxetine } \\
\mathrm{n}=17\end{array}$ & $P$ value \\
\hline \multirow[t]{3}{*}{ percentage of chemotherapy cycles with neuropathy (mean) } & Distal paresthesia & $84 \pm 25$ & $51 \pm 40$ & 0.01 \\
\hline & Throat discomfort & $69 \pm 37$ & $37 \pm 32$ & 0.01 \\
\hline & Cold induced dysesthesia & $79 \pm 30$ & $64 \pm 36$ & 0.13 \\
\hline
\end{tabular}

Table 5 Nerve conduction velocity 6 weeks after last Cycle of Chemotherapy

\begin{tabular}{|c|c|c|c|c|}
\hline & Group & $\mathbf{N}$ & Mean \pm SD & P Value \\
\hline \multirow[t]{3}{*}{ Right Deep Peroneal Nerve $\left(\mathrm{m} / \mathrm{s}^{\mathrm{a}}\right)$} & Placebo & 6 & $39.17 \pm 4.70$ & \multirow{4}{*}{0.04} \\
\hline & & & & \\
\hline & Duloxetin & 12 & $43.42 \pm 3.34$ & \\
\hline \multirow[t]{2}{*}{ Left Deep Peroneal Nerve (m/s) } & Placebo & 6 & $37.50 \pm 3.33$ & \\
\hline & Duloxetin & 12 & $42.67 \pm 4.25$ & 0.01 \\
\hline \multirow[t]{2}{*}{ Right Tibial Nerve (m/s) } & Placebo & 6 & $38.17 \pm 3.31$ & \multirow[b]{2}{*}{0.03} \\
\hline & Duloxetin & 12 & $42.58 \pm 4.83$ & \\
\hline \multirow[t]{2}{*}{ Left Tibial Nerve (m/s) } & Placebo & 6 & $38.67 \pm 3.93$ & \multirow[b]{2}{*}{0.14} \\
\hline & Duloxetin & 12 & $41.17 \pm 2.91$ & \\
\hline
\end{tabular}

a: meter per second

Table 6 Adverse effects (grade1-2) in placebo arm and duloxetine arm

\begin{tabular}{llll}
\hline Adverse Effects & Placebo $\mathrm{n}=15$ & Duloxetine $\mathrm{n}=17$ & P- value \\
\hline Nausea & $5(33 \%)$ & $9(53 \%)$ & 0.3 \\
Somnolence & $4(27 \%)$ & $8(47 \%)$ & 0.7 \\
Dry Mouth & $5(33 \%)$ & $7(41 \%)$ & 0.7 \\
Dizziness & $2(13 \%)$ & $7(41 \%)$ & 0.12 \\
Headache & $2(13 \%)$ & $4(23 \%)$ & 0.6 \\
Fatigue & $4(27 \%)$ & $3(18 \%)$ & 0.6 \\
\hline
\end{tabular}

Table 7 Nerve conduction velocity and Amplitude before Chemotheapy 


\begin{tabular}{|c|c|c|c|c|}
\hline & Group & $\mathrm{N}$ & Mean \pm SD & P-Value \\
\hline \multirow[t]{2}{*}{ Right Sural Nerve Amplitude } & Placebo & 6 & $14.63 \pm 1.69$ & \\
\hline & Duloxetin & 10 & $10.64 \pm 6.05$ & 0.43 \\
\hline \multirow[t]{2}{*}{ Left Sural Nerve Amplitude } & Placebo & 6 & $13.50 \pm 10.15$ & \\
\hline & Duloxetin & 10 & $10.88 \pm 6.17$ & 0.58 \\
\hline \multirow[t]{2}{*}{ Right Deep Peroneal Nerve Amplitude } & Placebo & 6 & $3.58 \pm 0.54$ & \\
\hline & Duloxetin & 12 & $3.76 \pm 1.42$ & 0.69 \\
\hline \multirow[t]{2}{*}{ Left Deep Peroneal Nerve Amplitude } & Placebo & 6 & $3.31 \pm 0.33$ & \\
\hline & Duloxetin & 12 & $4.04 \pm 2.65$ & 0.37 \\
\hline \multirow[t]{2}{*}{ Right Deep Peroneal Nerve Velocity } & Placebo & 6 & $40.50 \pm 1.64$ & \\
\hline & Duloxetin & 12 & $43.17 \pm 4.26$ & 0.07 \\
\hline \multirow[t]{2}{*}{ Left Deep Peroneal Nerve Velocity } & Placebo & 6 & $40.25 \pm 2.04$ & \\
\hline & Duloxetin & 12 & $42.81 \pm 3.87$ & 0.08 \\
\hline \multirow[t]{2}{*}{ Right Tibial Nerve Amplitude } & Placebo & 6 & $9.20 \pm 654$ & \\
\hline & Duloxetin & 12 & $7.76 \pm 3.26$ & 0.63 \\
\hline \multirow[t]{2}{*}{ Left Tibial Nerve Amplitude } & Placebo & 6 & $8.78 \pm 5.32$ & \\
\hline & Duloxetin & 12 & $7.48 \pm 2.98$ & 0.59 \\
\hline \multirow[t]{2}{*}{ Right Tibial Nerve Velocity } & Placebo & 6 & $39.67 \pm 2.06$ & \\
\hline & Duloxetin & 12 & $41.75 \pm 4.28$ & 0.18 \\
\hline \multirow[t]{2}{*}{ Left Tibial Nerve Velocity } & Placebo & 6 & $40.78 \pm 4.00$ & \\
\hline & Duloxetin & 12 & $41.08 \pm 4.10$ & 0.88 \\
\hline
\end{tabular}

Figures 


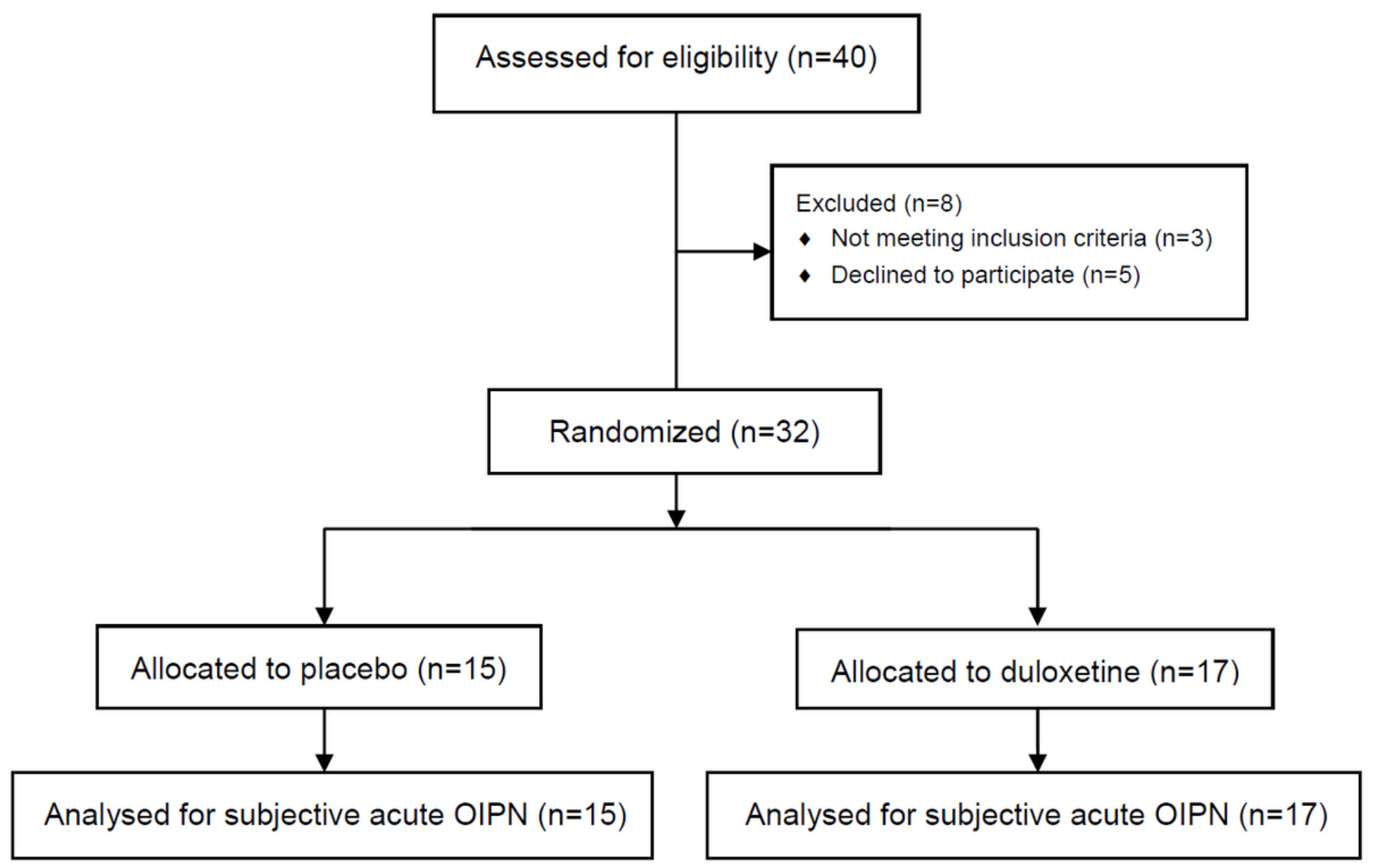

Figure 1

CONSORT diagram of the study

\section{Supplementary Files}

This is a list of supplementary files associated with this preprint. Click to download.

- SupplementalTable.docx 